\title{
REFLEXIONES SOBRE EL IMPACTO DEL COVID-19 EN LA LUCHA PARA LA ELIMINACIÓN DE LA VIOLENCIA CONTRA LA MUJER
}

\section{REFLECTIONS ON THE IMPACT OF COVID-19 IN THE STRUGGLE TO ELIMINATE VIOLENCE AGAINST WOMEN}

\author{
Karen Maribel Rebaza Vilchez ${ }^{1}$ \\ Ruth Antuaneth Buendia Casafranca ${ }^{2}$ \\ Martha Dayana Meléndez Muñoz ${ }^{3}$
}

1 Licenciada en Derecho por la Universidad Femenina del Sagrado Corazón (UNIFÉ). Magíster en Derecho Constitucional, Derechos Humanos y Gestión Pública con estudios de especialización en derecho internacional público. Docente fundadora del Círculo de Estudios de Derecho Constitucional y Derechos Humanos (CEDEC) de la UNIFÉ. Docente de la Facultad de Derecho y Ciencia Política de la Universidad Nacional Mayor de San Marcos (UNMSM) de los cursos: Sistema Interamericano de Derechos Humanos, Derecho Internacional Humanitario y Derecho de los Tratados.

${ }^{2}$ Estudiante del VI ciclo de la Facultad de Derecho de la Universidad Femenina del Sagrado Corazón (UNIFÉ). Coordinadora General del Círculo de Estudios de Derecho Constitucional y Derechos Humanos (CEDEC) de la misma casa de estudios. Licenciada en Ciencias de la Comunicación - UNIFÉ.

${ }^{3}$ Estudiante del VI ciclo de la Facultad de Derecho de la Universidad Femenina del Sagrado Corazón. Miembro del tópico de investigación del Círculo de Estudios de Derecho Constitucional y Derechos Humanos (CEDEC) de la misma casa de estudios. Miembro activo del Taller de Derecho Constitucional (TDC) de la Universidad Nacional Mayor de San Marcos (UNMSM).

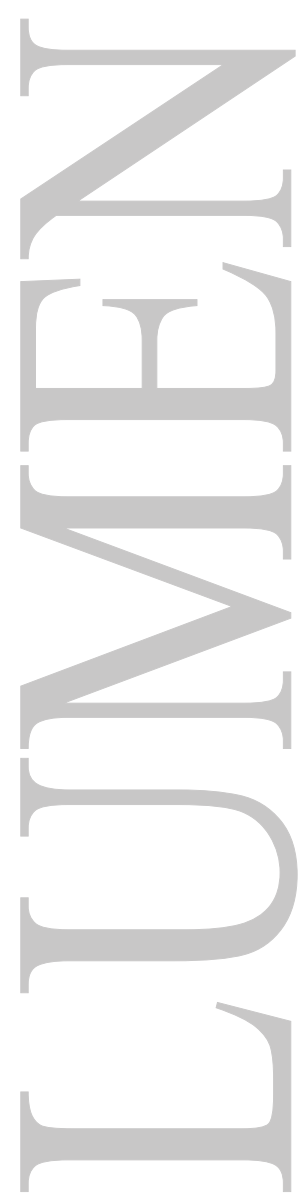




\title{
REFLEXIONES SOBRE EL IMPACTO DEL COVID-19 EN LA LUCHA PARA LA ELIMINACIÓN DE LA VIOLENCIA CONTRA LA MUJER
}

\author{
REFLECTIONS ON THE IMPACT OF COVID-19 IN THE STRUGGLE \\ TO ELIMINATE VIOLENCE AGAINST WOMEN
}

\author{
Karen Maribel Rebaza Vilchez \\ Ruth Antuaneth Buendia Casafranca \\ Martha Dayana Meléndez Muñoz
}

\begin{abstract}
RESUMEN:
La violencia contra la mujer es un problema social global que se ha visto agravado debido a la COVID-19 y las órdenes gubernamentales de confinamiento para evitar su propagación. Nuestro país, al igual que muchos de la región y el mundo, ha venido implementando políticas públicas destinadas a proteger a las mujeres contra el maltrato. Sin embargo, debido a este especial contexto, el impacto de dichas políticas se ha visto disminuido ya que muchas mujeres conviven con su agresor, en consecuencia, menos posibilidad de protección.

En ese contexto, el presente artículo tiene como objetivo evaluar, a la luz de los estándares internacionales en la materia, si nuestra legislación interna viene cumpliendo con su rol preventivo y protector o, por contrario, requiere cambios y/o ajustes para garantizar su eficacia en pandemia.
\end{abstract}

\section{PALABRAS CLAVE:}

Violencia contra la mujer, estado de emergencia, COVID-19, políticas públicas, obligación internacional.

\begin{abstract}
:
Violence against women is a global social problem that has been aggravated by COVID-19 and government confinement orders to prevent its spread. Our country, like many in the region and the world, has been implementing public policies designed to protect women against abuse. However, due to this special context, the impact of these policies has been diminished since many women live with their aggressor, consequently, have less possibility of protection.
\end{abstract}

In this context, this article aims to assess, in light of international standards on the matter, whether our internal law has been fulfilling its preventive and protective role or, on the contrary, wether requires changes or adjustments to guarantee its effectiveness. in pandemic.

\section{KEY WORDS:}

Violence against women, state of emergency, COVID-19, public policies, international obligation.

\section{INTRODUCCIÓN}

Semana tras semana, los medios de comunicación informan sobre nuevos casos de violencia contra la mujer en todas las regiones de nuestro país. Las medidas implementadas por el Gobierno para frenar el contagio de la COVID-19 trajo en sus primeros seis meses 18 víctimas de feminicidio, quince peruanas y tres venezolanas, según un informe del Observatorio de Criminalidad del Ministerio Publico.

Las relaciones entre mujeres y varones deben basarse en el respeto y la confianza, pero eso parece ser muy difícil de alcanzar. Según las cifras de la Encuesta Demográfica y de Salud Familiar (ENDES) realizada en el año 2018, el 63,2\% de mujeres entre 15 y 49 años han sido víctimas de algún tipo de violencia por parte de sus parejas sentimentales. 
La misma encuesta refleja cambios positivos pero también mucho trabajo pendiente. La cifra de violencia contra las mujeres, en el año 2009 , llegó a un $76,9 \%$, al año siguiente, 2010 , la data bajó a $75,8 \%$, en el año 2011 siguió el descenso a 74,2\%, en el año 2012, la cifra bajó una décima a $74,1 \%$, en el año 2013 se redujo a 73,5\%, en el año 2014 la cifra descendió a 72,4\%, en el año 2015 hablamos de 70,8\%, en el año 2016 llegó a 68,2\%, y en el año 2017 alcanzó el 65,4\%; sin embargo, dicha cifra se ha mantenido en los años siguientes y tendrá un aumento en el periodo 2020 producto de la pandemia.

Por años, como sociedad, hemos aceptado una realidad donde los roles establecidos para las mujeres era la de aceptar la violencia porque "es tu pareja", "porque seguro no lo atendiste bien", "porque no tenías la comida caliente", "porque no tenías que vestirte de esa forma", o "porque eras suya o de nadie". Lamentablemente, dichas frases han ido más allá del ámbito intrafamiliar y han sido mencionadas en algunos lugares que están destinados para la atención de las víctimas de violencia como las comisarías, juzgados, fiscalías, entre otros.

El reto es muy grande pues se deben romper esas barreras sociales enraizadas por décadas y desde la niñez. La violencia contra la mujer es un fenómeno desde lo social, porque le resta oportunidades a quien sufre directamente la violencia, pero también a quienes la sufren indirectamente: hijas, hijos, padres, madres, hermanas, hermanos, entre otros. Aún queda pendiente la atención integral a las hijas e hijos de las víctimas del último eslabón de la violencia contra las mujeres: el feminicidio.

El movimiento Manuela Ramos, en el documento que elevó al Congreso de la República antes del debate y aprobación de la modificación del Código Penal para que se incorpore el delito de feminicidio, recordaba que según la ENDES varones (INEI,2010), un 38,7\% de varones alguna vez casados o convivientes, considera que la violencia física hacia la mujer está justificada si ella es infiel, si descuida a los niños (12,9\%), si sale sin decirle a donde va $(8,3 \%)$, y si ella discute con él $(5,0 \%)$.

Diez años después, la realidad peruana no es muy distinta y lo muestra el Ministerio de la Mujer y Poblaciones Vulnerables (MIMP) en la cartilla elaborada contra los feminicidios bajo el título de "Alerta de Seguridad" donde se señala que al ser preguntados las y los jóvenes universitarias/os sobre la violencia contra las mujeres el $57 \%$ indica que le pegaría a su pareja si pierde el control, el $46 \%$ le pegaría a su pareja por alguna razón, el $45 \%$ le pegaría a su pareja levemente sin lastimarla y un $34 \%$ le pegaría a su pareja cuando ella tenga la culpa.

La pandemia ha creado un escenario mucho más desfavorable para las mujeres. Los casos de violencia reportados a los Centros de Emergencia Mujer del MIMP de enero a setiembre de este año han sumado 61,705 casos, de los cuales 52,752 son contra mujeres. En cuanto a tentativa de feminicidio o casos con características de feminicidio sumaron 186 y 96 casos respectivamente.

En ese contexto, en el presente artículo analizaremos tanto el desarrollo normativo internacional y nacional como los avances jurisprudenciales en relación a esta problemática para reflexionar sobre el impacto de la COVID-19, las buenas prácticas regionales para su tratamiento y los retos que enfrentamos.

\section{DESARROLLO NORMATIVO Y JURISPRUDENCIAL}

\subsection{Internacional}

Han transcurrido 38 años desde que en nuestro país entrará en vigencia la Convención sobre la eliminación de todas las formas de discriminación contra la mujer (CEDAW), aprobada por la Asamblea General de las Naciones Unidas mediante Resolución 34/180 del año 1979. 
Así, el Estado peruano se comprometió a asegurar "el pleno desarrollo y adelanto de la mujer, con el objeto de garantizar el ejercicio y el goce de los derechos humanos y las libertades fundamentales en igualdad de condiciones con el hombre" (art. 3).

La Convención además señala en su artículo 5 lo siguiente:

"Los Estados Partes tomarán todas las medidas apropiadas para:

a) Modificar los patrones socioculturales de conducta de hombres y mujeres, con miras a alcanzar la eliminación de los prejuicios y las prácticas consuetudinarias y de cualquier otra índole que estén basados en la idea de la inferioridad o superioridad de cualquiera de los sexos o en funciones estereotipadas de hombres y mujeres;

b) Garantizar que la educación familiar incluya una comprensión adecuada de la maternidad como función social y el reconocimiento de la responsabilidad común de hombres y mujeres en cuanto a la educación y al desarrollo de sus hijos, en la inteligencia de que el interés de los hijos constituirá la consideración primordial en todos los casos".

De otro lado, la Convención Interamericana para Prevenir, Sancionar y Erradicar la violencia contra la Mujer "Convención de Belém do Pará", adoptada en el vigésimo cuarto periodo ordinario de sesiones (1994) de la Asamblea General la Organización de Estados Americanos (OEA), vigente para el Perú desde el 4 de junio de 1996, en su artículo 1, precisa lo siguiente: "[...] debe entenderse por violencia contra la mujer cualquier acción o conducta, basada en su género, que cause muerte, daño o sufrimiento físico, sexual o psicológico a la mujer, tanto en el ámbito público como en el privado".

Asimismo, en su artículo 6 se señala que:

"El derecho de toda mujer a una vida libre de violencia incluye, entre otros:

a. el derecho de la mujer a ser libre de toda forma de discriminación, y

b. el derecho de la mujer a ser valorada y educada libre de patrones estereotipados de comportamiento y prácticas sociales y culturales basadas en conceptos de inferioridad o subordinación".

Sin embargo, pese a los años transcurridos y los compromisos internacionales asumidos a nivel universal e interamericano, se mantienen las desigualdades y la vulneración de los derechos humanos de las mujeres a nivel nacional, lo que nos muestra que es un problema estructural, como lo ha señalado la Comisión Interamericana de Derechos Humanos (CIDH) a través de un comunicado emitido en el mes de julio del año 2018, donde manifiesta su preocupación por la prevalencia de asesinatos y tentativas de asesinatos contra mujeres en nuestro país, a propósito del caso

En dicho comunicado, la CIDH llama al Estado a implementar estrategias integrales para prevenir estos hechos, para cumplir con su obligación de investigar, juzgar y sancionar a los responsables, y para ofrecer protección y reparación integral a todas las víctimas. Asimismo, lo exhorta a adoptar medidas inmediatas y urgentes para prevenir, investigar, juzgar, sancionar y reparar todo acto de violencia contra las mujeres; lo que incluye medidas integrales elaboradas con la participación de las mujeres beneficiarias.

Resulta importante precisar que, en el mes de la emisión del comunicado, en el país se habían suscitado lamentables hechos de violencia; uno de ellos el caso de Eyvi Ágreda Marchena quien 
fuera víctima de violencia por parte de su compañero de trabajo quien le roció un líquido inflamable al cuerpo y producto de la quemadura falleció. En el mismo mes, Gilda Branz Sol Mujica fue atacada con ácido, violada y apuñalada por su agresor.

Ahora bien, la CIDH emitió el Informe $N^{\circ} 54 / 01$ frente al Caso $N^{\circ} 12.051$. María da Penha Fernandes, en el cual consideró que las condiciones de violencia doméstica que sufrió fueron toleradas por el Estado brasileño y que, por lo tanto, existió responsabilidad del Estado por el incumplimiento de las obligaciones derivadas de la Convención de Belém do Pará al no sancionar al victimario durante quince años pese a los reclamos oportunamente efectuados.

Entre las recomendaciones dadas al Estado brasileño se encuentran las siguientes:

"1. Completar rápida y efectivamente el procesamiento penal del responsable de la agresión y tentativa de homicidio en perjuicio de la señora Maria da Penha Fernandes Maia.

2. Llevar igualmente a cabo una investigación seria, imparcial y exhaustiva para determinar la responsabilidad por irregularidades o retardos injustificados que impidieron el procesamiento rápido y efectivo del responsable; y tomar las medidas administrativas, legislativas y judiciales correspondientes.

3. Adoptar, sin perjuicio de las eventuales acciones contra el responsable civil de la agresión, medidas necesarias para que el Estado asigne a la víctima adecuada reparación simbólica y material por las violaciones aquí establecidas, en particular su falla en ofrecer un recurso rápido y efectivo; por mantener el caso en la impunidad por más de quince años; y por evitar con ese retraso la posibilidad oportuna de acción de reparación e indemnización civil".

Como se puede observar, la CIDH en su informe señaló que el Estado debía completar el proceso penal iniciado contra su agresor y determinar las responsabilidades correspondientes. En el 2002 el proceso fue concluido en el fuero nacional brasileño, por ello, el caso no llego a ser sometido a la Corte Interamericana de Derechos Humanos (Corte IDH).

Otro caso importante visto en el sistema interamericano, es el de González y otras (Campo Algodonero) vs. México, cuya sentencia expedida por la Corte IDH, el 16 de noviembre del 2009, reconoce como factor de violencia contra las mujeres el incremento de su empleabilidad en la industria del maquillaje en Ciudad de Juárez a partir de 1993 luego de la suscripción del Tratado de Libre Comercio con América del Norte:

“(...) el aumento de la preferencia laboral en la contratación de mujeres, "las maquilladoras causaron cambios en la vida laboral de éstas, lo cual impactó también su vida familiar porque los roles tradicionales empezaron a modificarse, al ser la mujer la proveedora del hogar".

En el citado caso, la Corte IDH tomó en cuenta el reconocimiento del Estado de la situación de violencia contra la mujer en Ciudad Juárez, así como la influencia de la cultura de discriminación hacia la mujer observada en los informes de la Relatoría de la CIDH, del CEDAW y de Amnistía Internacional.

De la lectura de dicha sentencia queda claro que los Estados deben generar medidas eficientes para la prevención, investigación y sanción de casos de violencia contra las mujeres; además de contar con un marco legal eficaz para que las denuncias no queden en la impunidad que es lo que genera mayor vulneración a las víctimas y sus familiares e incluso a la sociedad.

Otros casos importantes ante la CIDH sobre vulneración a los derechos humanos de las mujeres son los siguientes: 


\begin{tabular}{|c|c|c|}
\hline Caso & Recomendaciones CIDH al Estado & Estado de Cumplimiento \\
\hline 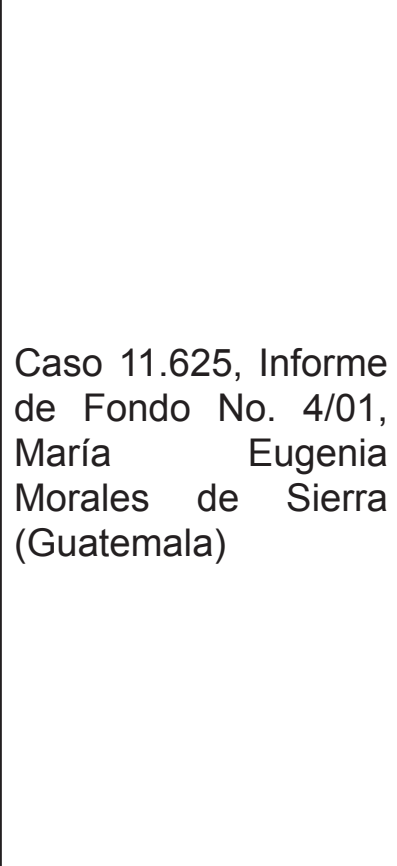 & $\begin{array}{l}\text { 1. Adecuar las disposiciones pertinentes } \\
\text { del Código Civil para equilibrar el } \\
\text { reconocimiento jurídico de los deberes } \\
\text { recíprocos de la mujer y del hombre } \\
\text { dentro del matrimonio, y adoptar las } \\
\text { medidas legislativas y de otra índole } \\
\text { necesarias para reformar el artículo } \\
317 \text { del Código Civil, para hacer } \\
\text { congruente la legislación nacional } \\
\text { con las normas de la Convención } \\
\text { Americana y dar efecto pleno a los } \\
\text { derechos y libertades que la misma } \\
\text { garantiza a María Eugenia Morales } \\
\text { de Sierra. } \\
\text { 2. Reparare indemnizar adecuadamente } \\
\text { a María Eugenia Morales de Sierra } \\
\text { por los daños ocasionados por } \\
\text { las violaciones establecidas en el } \\
\text { presente Informe. }\end{array}$ & PARCIAL \\
\hline $\begin{array}{l}\text { Caso 12.626, Informe } \\
\text { de Fondo No. 80/11, } \\
\text { Jessica Lenahan } \\
\text { (Gonzales) y otros } \\
\text { (Estados Unidos) }\end{array}$ & $\begin{array}{l}\text { 1. Emprender una investigación seria, } \\
\text { imparcial y exhaustiva con el objetivo } \\
\text { de determinar la causa, hora y lugar } \\
\text { de las muertes de Leslie, Katheryn } \\
\text { y Rebecca Gonzales, e informar } \\
\text { debidamente a sus familiares del } \\
\text { curso de la investigación. } \\
\text { 2. Realizar una investigación seria, } \\
\text { imparcial y exhaustiva de las fallas } \\
\text { sistémicas que ocurrieron en relación } \\
\text { con la ejecución de la orden de } \\
\text { protección de Jessica Lenahan como } \\
\text { garantía de no repetición, incluyendo } \\
\text { una investigación para determinar las } \\
\text { responsabilidades de los funcionarios } \\
\text { públicos por violar la legislación del } \\
\text { estado y/o federal, y sancionar a los } \\
\text { responsables. } \\
\text { 3. Ofrecer una plena reparación a } \\
\text { Jessica Lenahan y a sus familiares, } \\
\text { considerando su perspectiva y } \\
\text { necesidades específicas. }\end{array}$ & PARCIAL \\
\hline
\end{tabular}


Caso 12.551, Informe de Fondo No. 51/13, Paloma Angélica Escobar Ledezma y otros (México)
1. Completar la investigación de manera oportuna, inmediata, seria e imparcial con el objeto de esclarecer el asesinato de Paloma Angélica Escobar e identificar, juzgar y, en su caso sancionar a los responsables.

2. Reparar plenamente a los familiares de Paloma Angélica Escobar por las violaciones de los derechos humanos aquí establecidas.

3. Implementar, como medida de no repetición, una política estatal integral y coordinada, respaldada con recursos públicos adecuados, para garantizar que los casos específicos de violencia contra las mujeres, sean adecuadamente prevenidos, investigados, sancionados y reparados en la Ciudad de Chihuahua.

Elaboración propia.

Fuente: Anexo 2 del Informe "Impactos de casos de discriminación y violencia contra mujeres, niñas y adolescentes" elaborado por la Comisión Interamericana de Derechos Humanos.

La Corte IDH también en su cuadernillo de Jurisprudencia N 4 "Derechos Humanos y Mujeres en su capítulo Violencia de Género y Violencia Sexual" hizo importantes precisiones relativas al tema desde la perspectiva de la Convención Americana de Derechos Humanos (CADH) y la Convención Belém do Pará, bajo el siguiente detalle:

\begin{tabular}{|c|c|}
\hline CASO & PÁRRAFOS MÁS IMPORTANTES DE LA SENTENCIA \\
\hline $\begin{array}{l}\text { Caso Fernández Ortega y } \\
\text { otros Vs. México. Excepción } \\
\text { Preliminar, Fondo, } \\
\text { Reparaciones y Costas. } \\
\text { Sentencia de } 30 \text { de agosto } \\
\text { de } 2010 .\end{array}$ & $\begin{array}{l}\text { 100. En primer lugar, a la Corte le resulta evidente que la violación } \\
\text { sexual es untipo particular de agresión que, en general, se caracteriza } \\
\text { por producirse en ausencia de otras personas más allá de la víctima } \\
\text { y el agresor o los agresores. Dada la naturaleza de esta forma de } \\
\text { violencia, no se puede esperar la existencia de pruebas gráficas o } \\
\text { documentales y, } \\
\text { por ello, la declaración de la víctima constituye una prueba } \\
\text { fundamental sobre el hecho. } \\
\text { 118. Este Tribunal recuerda, como lo señala la Convención de } \\
\text { Belém do Pará, que la violencia contra la mujer no sólo constituye } \\
\text { una violación de los derechos humanos, sino que es "una ofensa } \\
\text { a la dignidad humana y una manifestación de las relaciones de } \\
\text { poder históricamente desiguales entre mujeres y hombres", que } \\
\text { "trasciende todos los sectores de la sociedad independientemente } \\
\text { de su clase, raza o grupo étnico, nivel de ingresos, cultura, nivel } \\
\text { educacional, edad o } \\
\text { religión y afecta negativamente sus propias bases". }\end{array}$ \\
\hline
\end{tabular}


Caso Veliz Franco y otros Vs. Guatemala. Excepciones Preliminares, Fondo, Reparaciones y Costas. Sentencia de 19 de mayo de 2014.
178. Este Tribunal ya ha determinado que si bien no puede asegurarse que todos los homicidios de mujeres sucedidos en la época de los hechos fueran por razones de género, resulta verosímil que el de María Isabel si lo fuera, de acuerdo a cómo se encontró el cuerpo de la niña. En efecto, se ha indicado que las mujeres víctimas de homicidios por razones de género con frecuencia presentaban signos de brutalidad en la violencia ejercida contra ellas, así como signos de violencia sexual o la mutilación de los cuerpos [...].

De forma acorde a tales características, el cadáver de María Isabel fue encontrado con evidentes signos de violencia, inclusive señales de ahorcamiento, una herida en el cráneo, una cortadura en la oreja y mordiscos en las extremidades superiores; su cabeza estaba envuelta por toallas y una bolsa, y tenía alimentos en su boca y su nariz [...], además, la blusa y el bloomer que llevaba estaban rotos en la parte inferior $[\ldots]$.

Ello resulta relevante y suficiente a los efectos de la aplicación al caso del artículo 7 de la Convención de Belém do Pará. Interesa aclarar que la falta de certeza absoluta sobre lo expresado se vincula a la falta de conclusión de la investigación interna, así como al modo en que ésta hasta ahora se ha desarrollado. Así, por ejemplo, elementos trascendentes como la presencia de violencia sexual en los hechos no han sido determinados en una forma certera [...].

207. La Corte estima que la violencia basada en el género, es decir la violencia dirigida contra una mujer por ser mujer o la violencia que afecta a la mujer de manera desproporcionada, es una forma de discriminación en contra de la mujer, tal como han señalado otros organismos internacionales de protección de derechos humanos, como el Tribunal Europeo de Derechos Humanos y el CEDAW.

Tanto la Convención de Belém do Pará (preámbulo y artículo 6) como el CEDAW (preámbulo) han reconocido el vínculo existente entre la violencia contra las mujeres y la discriminación. En el mismo sentido, el Convenio del Consejo de Europa sobre prevención y lucha contra la violencia contra las mujeres y la violencia doméstica (Estambul, 2011) afirma que "la violencia contra las mujeres es una manifestación de desequilibrio histórico entre la mujer y el hombre que ha llevado a la dominación y a la discriminación de la mujer por el hombre, privando así a la mujer de su plena emancipación", así como que "la naturaleza estructural de la violencia contra las mujeres está basada en el género".

Elaboración Propia.

Fuente: Cuadernillo de Jurisprudencia $N^{\circ}$ 4: Derechos Humanos y Mujeres en su capítulo Violencia de Género y Violencia Sexual. Corte Interamericana de Derechos Humanos. 


\subsection{Nacional}

La Ley $\mathrm{N}^{\circ} 30364$, Ley para prevenir, sancionar y erradicar la violencia contra la mujer y los integrantes del grupo familiar, en el artículo 5, define la violencia contra la mujer con lo siguiente:

La violencia contra las mujeres es cualquier acción o conducta que les causa muerte, daño o sufrimiento físico sexual o psicológico por su condición de tales, tanto en el ámbito público como en el privado.

Se entiende como violencia contra la mujeres:

a. La que tenga lugar dentro de la familia o unidad doméstica o en cualquier otra relación interpersonal, ya sea que el agresor comparta o haya compartido el mismo domicilio que la mujer. Comprende, entre otros, violación, maltrato físico o psicológico y abuso sexual. b. La que tenga en la comunidad, sea perpetrada por cualquier persona y comprende, entre otros, violación, abuso sexual, tortura, trata de personas, prostitución forzada, secuestro y acoso sexual en el lugar de trabajo, así como en instituciones educativas, establecimientos de salud o cualquier otro lugar.

c. La que sea perpetrada o tolerada por los agentes del Estado donde quiera que ocurra.

\section{Elaboración propia.}

Fuente: La Ley $N^{\circ} 30364$, Ley para prevenir, sancionar y erradicar la violencia contra la mujer y los integrantes del grupo familiar

En la misma línea, el Tribunal Constitucional refirió que la violencia contra la mujer es un tipo de violencia basada en el género, basada en manifestaciones de las relaciones de poder históricamente desiguales entre mujeres y hombre, la cual ha venido ocasionando una grave vulneración a la dignidad humana (STC Exp. Nº 03378-2019-PA/TC, fundamento. 54).

Esta forma de violencia está apoyada en concepciones y costumbres interiorizadas; es decir, en ideas de inferioridad y subordinación de las mujeres frente a la superioridad y poder de los hombres. En relación a ello, el MIMP en el documento "Violencia basada en género. Marco conceptual para las políticas públicas y la acción del Estado" señala que la violencia basada en el género es entendida como:

"Cualquier acción o conducta, basada en el género y agravada por la discriminación proveniente de la coexistencia de diversas identidades (raza, clase, identidad sexual, edad, pertenencia étnica, entre otras), que causa muerte, daño o sufrimiento físico, sexual o psicológico a una persona, tanto en el ámbito público como en el privado. Se trata de aquella violencia que ocurre en un contexto de discriminación sistemática contra la mujer y contra aquellos que confrontan el sistema de género, sea al interior de las familias o fuera de ellas, al margen de su sexo, que no se refiere a casos aislados, esporádicos o episódicos de violencia, sino que están referidos al sistema de género imperante, que remite a una situación estructural y a un fenómeno social y cultural enraizado en las costumbres y mentalidades de todas las sociedades y que se apoya en concepciones referentes a la inferioridad y subordinación de las mujeres y la supremacía y poder de los varones". (p. 23)

En razón de ello, se han desarrollado diferentes políticas públicas tendientes a garantizar que las mujeres tengan una vida libre de violencia desde un enfoque integral e intersectorial, reconociendo al problema como de índole social, político, económico y de salud pública. 
El Programa Nacional Contra la Violencia Familiar y Sexual (PNCVFS) es el órgano encargado de diseñar y ejecutar a nivel nacional acciones y políticas de prevención, atención y apoyo a las personas involucradas en hechos de violencia familiar y/o sexual, contribuyendo así a mejorar la calidad de vida de la población, desde una perspectiva de género.

En un primer momento, dicho Programa estuvo enmarcado en el Plan Nacional Contra la Violencia hacia la Mujer 2009 - 2015, aprobado en el año 2009 mediante Decreto Supremo $N^{\circ}$ 003-2009-MIMDES, el cual planteaba el desarrollo de la mujer en condiciones de igualdad sin ningún tipo de distinción y la eliminación de patrones socio-culturales discriminatorios.

En su ejecución se dio la participación de gobiernos regionales y locales y de diversos sectores del Poder Ejecutivo, tales como el Ministerio del Interior, Ministerio de Salud, Ministerio de Educación, Ministerio de Justicia -hoy Ministerio de Justicia y Derechos Humanos-, Ministerio de Relaciones Exteriores, Ministerio Público (miembros supernumerarios), Poder Judicial (miembros supernumerarios). Asimismo, incorporaba el valioso apoyo de la Defensoría del Pueblo (miembros supernumerarios).

Así, se reforzó la convicción de que, para alcanzar sólidos y fructíferos cambios en nuestra nación, necesitamos garantizar el acceso a las mujeres a servicios públicos de calidad, especializados en la atención de víctimas de violencia de género mediante los cuales no solo se eviten los estereotipos de género que perpetúan la violencia, sino que se alcancen resultados concretos.

El Plan Nacional Contra la Violencia hacia la Mujer 2009 - 2015, tuvo vigencia hasta el mes de diciembre del año 2015. Una vez terminada su vigor se promulga la Ley $\mathrm{N}^{\circ} 30364$, Ley para prevenir, sancionar y erradicar la violencia contra las mujeres y los integrantes del grupo familiar, publicada el 23 de noviembre de 2015.

La referida norma establece la creación del Sistema Nacional para la Prevención, Sanción y Erradicación de la Violencia contra las Mujeres y los Integrantes del Grupo Familiar, como un sistema funcional y ello define el estatus que adopta este nuevo plan nacional, como Plan Especial Multisectorial.

Transcurrido siete meses, en julio del año 2016, mediante Decreto Supremo N 008-2016- MIMP se aprobó el Plan Nacional Contra la Violencia de Género 2016 - 2021.

\begin{tabular}{|c|c|}
\hline VISIÓN & MISIÓN \\
\hline $\begin{array}{l}\text { El Perú es una sociedad donde se han } \\
\text { eliminado los patrones socioculturales } \\
\text { discriminatorios y se han establecido } \\
\text { relaciones de igualdad en el ejercicio de } \\
\text { derechos y relaciones libres de violencia por } \\
\text { razones de género, raza, etnia, clase social, } \\
\text { edad, orientación sexual, garantizando los } \\
\text { derechos humanos de todas las personas y } \\
\text { en especial de las que están en situación de } \\
\text { vulnerabilidad. }\end{array}$ & $\begin{array}{l}\text { Desde el Estado, se adoptan e implementan } \\
\text { políticas públicas integrales para la prevención, } \\
\text { atención, protección, rehabilitación de las } \\
\text { personas afectadas y la sanción, reeducación } \\
\text { de las personas agresoras, que transversalizan } \\
\text { los enfoques de género, derechos humanos, } \\
\text { intercultural, de integralidad, de interseccionalidad } \\
\text { y generacional, en el sistema educativo formal y } \\
\text { en las familias, para la prevención de la violencia } \\
\text { de género; en la prestación de servicios públicos } \\
\text { de calidad, a nivel intersectorial, interinstitucional } \\
\text { e intergubernamental y en el sistema de justicia, } \\
\text { para facilitar el acceso oportuno a una justicia } \\
\text { efectiva que garantice el derecho a una vida libre } \\
\text { de violencia. }\end{array}$ \\
\hline
\end{tabular}

Elaboración propia 
Luego de dos planes nacionales entre los períodos 2002-2007 y 2009-2015, y próximos a cumplir el período de vigencia de un tercero, aún la deuda está pendiente frente a la ardua tarea por evitar casos de violencia hacia las mujeres diariamente en el país.

Se necesitan compromisos claros y precisos para mejorar la articulación interinstitucional e intergubernamental para enfrentar el complejo problema de la violencia de género.

\section{POLÍTICAS PÚBLICAS INTERNAS EN LA LUCHA CONTRA LA VIOLENCIA A LA MUJER EN CONTEXTO DE PANDEMIA}

El pasado 15 de marzo, mediante Decreto Supremo N 044-2020-PCM, el Gobierno peruano declaró Estado de Emergencia a nivel nacional y dispuso medidas de aislamiento social obligatorio por el brote del COVID-19, pandemia que ha dejado hasta la fecha más de cinco millones de muertos a nivel mundial. Dichas medidas pusieron en evidencia otra pandemia que afecta a nuestro país y ha ido en incremento, la violencia contra la mujer.

En ese contexto, el MIMP ha emitido diferentes políticas destinadas a prevenir los casos de violencia contra la mujer, las cuales son las siguientes:

\begin{tabular}{|c|c|}
\hline $\begin{array}{l}\text { Servicios de atención a } \\
\text { víctimas de violencia se } \\
\text { activarán durante estado de } \\
\text { emergencia. }\end{array}$ & $\begin{array}{l}\text { Dispuso que sus servicios Línea } 100 \text { y Hogares de Refugio } \\
\text { Temporal del MIMP, comprendidos como esenciales por el Decreto } \\
\text { de Urgencia 044-2020-PCM, continúen brindando atención con el } \\
\text { objetivo de garantizar la protección de los derechos de las mujeres } \\
\text { víctimas de violencia. }\end{array}$ \\
\hline $\begin{array}{l}\text { Implementación estrategia } \\
\text { de acompañamiento } \\
\text { psicológico telefónico para } \\
\text { prevenir la violencia durante } \\
\text { el aislamiento social }\end{array}$ & $\begin{array}{l}\text { A partir del } 26 \text { de marzo de } 2020 \text { se implementó la Estrategia de } \\
\text { Acompañamiento Psicológico Telefónico, a cargo de psicólogos y } \\
\text { psicoterapeutas que brindarán atención profesional y oportuna para } \\
\text { prevenir la violencia en mujeres adultas y el ejercicio de conductas } \\
\text { violentas en el caso de hombres, que se encuentran en aislamiento } \\
\text { social. }\end{array}$ \\
\hline $\begin{array}{l}\text { Ampliación en la atención } \\
\text { del chat } 100 \text { para orientar } \\
\text { y brindar consejería a } \\
\text { varones a fin de prevenir la } \\
\text { violencia familiar durante la } \\
\text { cuarentena. }\end{array}$ & $\begin{array}{l}\text { Ante esta coyuntura, el Ministerio de la Mujer y Poblaciones } \\
\text { Vulnerables, a través del Programa Nacional para la Prevención } \\
\text { y Erradicación de la Violencia contra las Mujeres e Integrantes } \\
\text { del Grupo Familiar - Aurora, amplió el servicio preventivo de la } \\
\text { plataforma virtual Chat } 100 \text {, con el fin de orientar a los varones } \\
\text { mayores de } 18 \text { años durante la cuarentena. } \\
\text { En ese sentido, los especialistas de la intervención preventiva } \\
\text { "Hombres por la igualdad" proporcionarán orientación y consejería } \\
\text { las } 24 \text { horas del día y los } 7 \text { días de la semana sobre cómo solucionar } \\
\text { problemas de pareja, mejorar las relaciones con los hijos e hijas y } \\
\text { fomentar una paternidad corresponsable y activa a fin de prevenir } \\
\text { situación de conflicto o violencia y promover relaciones igualitarias } \\
\text { en el hogar. }\end{array}$ \\
\hline
\end{tabular}

Elaboración propia. 
Al cierre de este artículo una joven de 21 años fue presuntamente agredida sexualmente por cinco jóvenes en la ciudad de Lima; el abogado de uno de ellos señaló a diversos medios de comunicación que "... a la señorita le gustaba la vida social". Como se puede observar, dichas estrategias son importantes pero insuficientes.

\section{BUENAS PRÁCTICAS ESTATALES REGIONALES}

En el presente apartado describiremos las políticas que han venido aplicando diferentes países vecinos con la finalidad de prevenir y erradicar la violencia contra la mujer durante la pandemia.

\section{Argentina}

En seguimiento a la expedición de la Ley № 27.541, que declara en emergencia pública en materia económica, financiera, fiscal, administrativa, etc. por la propagación del COVID-19, el Ministerio de las Mujeres, Géneros y Diversidad dispuso las siguientes políticas para frenar el aumento de violencia a la mujer:

\section{- Asistencia rápida ante situaciones de violencia}

o Mediante la Línea 144 se atiende, contiene y asesora integralmente a las personas en situación de violencia por motivos de género, sus familiares y su círculo de confianza. Esta línea, que ha recibido un reforzamiento presupuestal durante la pandemia, es de alcance federal y se ha vuelto indispensable en el marco de la emergencia sanitaria.

- Mediante la dirección electrónica: linea144@mingeneros.gob.ar, cuentan con un canal de comunicación gratuita complementaria a la Línea 144 para recibir contención y asesoramiento ante situaciones de violencia por motivos de género.

- Se habilitó una línea de contacto directo a través del WhatsApp para aquellas personas que no puedan llamar por teléfono: 1127716463.

- Se dispuso a nivel ministerial que las personas en situación de violencia por motivos de género pueden estar exceptuadas de las medidas de aislamiento en casos de fuerza mayor, cuando requieran pedir asistencia o realizar una denuncia.

- Se facilitó canales de comunicación entre los gobiernos provinciales y/o locales con las organizaciones civiles para que puedan contactarse directamente y solicitar recursos para la atención de casos de violencia.

\section{- Asistencia integral interinstitucional ante situaciones de violencia}

- Se formó un Comité de Seguimiento de la situación de las mujeres y personas LGBTI+ en situación de violencia por motivos de género con la finalidad de que los dispositivos de gestión de la Línea 144 en esas jurisdicciones permanezcan vigentes durante el aislamiento social preventivo y obligatorio.

- Se gestionó con el Ministerio de Desarrollo Social de la Nación la incorporación de mujeres en situación de violencia por motivos de género al programa Potenciar Trabajo para acompañarlas desde la asistencia social y económica.

- Se solicitó al Poder Judicial de la Nación y los poderes judiciales provinciales la prórroga automática de las medidas de protección judiciales que podían vencer durante la cuarentena. 
- Se lanzó con apoyo de la Confederación Farmacéutica Argentina una campaña solidaria para poner a disposición las farmacias como espacios donde las mujeres en situación de violencia puedan acudir para solicitar asistencia y ponerse en contacto directo con la Línea 144. A través de la iniciativa "Barbijo Rojo", se distribuyó una guía de actuación con pautas y criterios para que los farmacéuticos puedan actuar ante la solicitud de personas en situación de violencia por motivos de género frente a la dificultad de las personas que en la cuarentena conviven con sus agresores.

- Se participa activamente a nivel internacional en las reuniones organizadas por la Comisión Interamericana de Mujeres y el Banco Interamericano de Desarrollo. La ministra de las Mujeres, Géneros y Diversidad, Elizabeth Gómez Alcorta, participó de la Reunión de Altas Autoridades de Género de la Región: desafíos, implicaciones y el rol de los mecanismos para el adelanto de las mujeres ante la pandemia del COVID19, como una plataforma de apoyo internacional para el fortalecimiento de los lazos solidarios para la prevención y asistencia de situaciones de violencia por motivos de género.

- Se lanzó la campaña de comunicación \#CuarentenaEnRedes, mediante la cual se concientiza a las personas sobre la importancia de ser empáticos y solidarios siempre, pero sobre todo en este contexto de emergencia y crisis sanitaria.

\section{Paraguay}

Se presentó el Plan "Paraguay protege a las mujeres y a los niños, niñas y adolescentes contra la violencia, en el contexto de la emergencia por Covid-19", el cual tiene con la finalidad de fortalecer las acciones de respuesta del país ante la problemática de la protección de mujeres y de niños, niñas y adolescentes contra la violencia, en el contexto de la emergencia por Covid-19.

Dicho Plan incluye servicios de atención urbana y rural, con articulación interinstitucional, con apoyo de la sociedad civil y el sector privado. Asimismo, contiene la difusión de material informativo en idioma castellano y guaraní sobre los mecanismos de atención existentes en el contexto de la emergencia por Covid-19.

Al respecto, es importante indicar que dicho Plan está siendo difundida mediante un campaña de comunicación apoyada por una alianza multisectorial compuesta por agencias de la ONU, ministerios del Gabinete Social, grupos privados de medios de comunicación, compañías de telecomunicaciones y plataformas de medios sociales.

\section{$\underline{\text { Chile }}$}

El Ministerio de la Mujer y la Equidad de Género emitió el "Plan de Contingencia de atención, protección y reparación ante los efectos del coronavirus" que tiene como objetivo:

o Velar por la continuidad de la atención, protección y reparación de mujeres víctimas y posibles víctimas de violencia en la red del Servicio Nacional de la Mujer y Equidad de Género (SernamEG) en sus distintas instancias, así como, proteger a los funcionarios que operan dichos servicios.

- Difundir material de uso público a través de diversos canales de comunicación gubernamentales u otros que llegue a las mujeres que puedan o estén en riesgo de sufrir violencia.

o Continuidad y fortalecimiento de atención en Centros de la Mujer y Centros Violencia Sexual. 
- Continuidad de atención en Casas de Acogida mediante la coordinación entre la Secretaría Regional Ministerial (SEREMI) de la Mujer y el SEREMI de Salud para eventuales casos de sospecha de contagio o contagio positivo de Covid-19 en mujeres y sus hijas e hijos que residan en Casa de Acogida así como también respecto del equipo que trabaja en ellas. Las Casas de Acogida fueron declaradas dentro de los "Servicios de Utilidad Pública", por lo que se ha instruido formalmente la continuidad de estos servicios, con sus protocolos y lineamientos específicos en pandemia.

o Continuidad de atención casos de violencia extrema.

o Coordinación permanente del Circuito Intersectorial de Femicidio (CIF) para la óptima atención de mujeres.

o Fono de Atención \#1455 (Orientación e Información en Violencia).

o Lanzamiento de la clave de atención "Mascarilla 19" en las farmacias. Su funcionamiento consiste en que si una mujer dice dicha clave en una farmacia el personal que atiende sabrá inmediatamente que se trata de una situación de violencia, le pedirá sus datos personales y luego llamarán al Fono 1455 de Orientación de Violencia contra la Mujer y en un caso más grave con el Fono 149 de Carabineros.

o Creación de canales de comunicación silenciosa. WhatsApp +569 97007000 y Chat Web 1455.

\section{CONCLUSIONES}

- Los patrones socioculturales vigentes en la sociedad naturalizan, promueven y perpetúan la violencia contra las mujeres. Ello debe ser eliminado progresivamente mediante legislación y políticas públicas adecuadas que estén elaboradas teniendo en cuenta como eje central dicha problemática.

- En el sistema interamericano de derechos humanos, la Comisión y Corte Interamericana han exhortado constantemente a los Estados a cumplir con las obligaciones generadas por la Convención Interamericana para Prevenir, Sancionar y Erradicar la violencia contra la Mujer "Convención de Belém do Pará con el objetivo de promover una vida libre de violencia y evitar una futura responsabilidad internacional ante su incumplimiento.

o En los últimos diez años, el Estado peruano ha demostrado mayor interés en generar leyes y políticas públicas para la lucha contra la violencia a la mujer a la luz de los instrumentos internacionales ratificados en la materia. Pese a ello, los últimos tres años no se ha demostrado un descenso en las cifras, lo que lleva a iniciar nuevas estrategias que tengan en cuenta elementos como el confinamiento producto de la pandemia.

- Diversos países de la región han adoptado medidas y/o políticas estatales especiales para brindar ayuda y apoyo a las mujeres víctimas de violencia. Como elemento común se ha detectado el uso y provecho de las nuevas tecnologías de la información toda vez que están al alcance de todos. 


\section{RECOMENDACIONES}

- Iniciar una campaña de sensibilización sostenida en los medios de comunicación sobre los derechos humanos de las mujeres y los tipos de violencia a los que son sujetas y deben ser erradicadas.

- Promover el contenido de los instrumentos internacionales ratificados por nuestro país entre los jueces, fiscales, y personal policial, para una mejor garantía de los derechos de las mujeres teniendo en cuenta que son el primer contacto con dicha problemática.

- Buscar el apoyo de la sociedad civil y el sector privado en la difusión de los mecanismos de apoyo a las mujeres víctimas de violencia para su real difusión e impacto. Para ello deben habilitarse mayores canales de atención virtual y telefónica, teniendo como partida que, solo en casos de emergencia, debe ser preferida la atención presencial.

- Involucrar a los estudiantes de las facultades de Derecho en las actividades de atención y prevención de la violencia iniciados por el Ministerio de la Mujer y Poblaciones Vulnerables para que se formen adecuadamente en la materia y eleven su nivel de conciencia sobre la problemática.

o El Instituto Nacional de Estadística e Informática (INEI) en conjunto con el Ministerio de la Mujer y Poblaciones Vulnerables (MIMP), debe generar nueva data estadística teniendo en cuenta entre sus indicadores las nuevas circunstancias generadas por la pandemia de la COVID-19.

\section{REFERENCIAS}

- Centro por la Justicia y el Derecho Internacional - CEJIL. (24 de junio de 2019). México: Ante la ausencia de reparación y justicia, se presenta caso de Marisela Escobedo ante la CIDH. Recuperado de: https://cejil.org/es/mexico-ausencia-reparacion-y-justicia-se- presenta-casomarisela-escobedo-cidh

- Congreso de la República. (2002). Plan Nacional contra la Violencia hacia La Mujer 2002 2007. Recuperado de: http://www4.congreso.gob.pe/historico/cip/materiales/vfamiliar/ plannacional2002_2007.pdf

- Congreso de la República. (6 de noviembre de 2015). Ley № 30364, Ley para prevenir, sancionar y erradicar la violencia contra la mujer y los integrantes del grupo familiar. Recuperado de: https://busquedas.elperuano.pe/normaslegales/ley-para-prevenir-sancionary-erradicar-la-violencia-contra-ley-n-30364-1314999-1/

- Comisión Interamericana de Derechos Humanos (2019). Violencia y discriminación contra mujeres, niñas y adolescentes: Buenas prácticas y desafíos en América Latina y en el Caribe. Recuperado de: https://www.oas.org/es/cidh/informes/pdfs/ViolenciaMujeresNNA. pdf

- Comisión Interamericana de Derechos Humanos (2001). Informe $N^{\circ}$ 54/01, caso María da Penha Maia Fernades vs. Brasil. Recuperado de: https://www.cidh.oas.org/ annualrep/2000sp/Capitulolll/Fondo/Brasil12.051.htm

- Comisión Interamericana de Derechos Humanos (2018). Comunicado de prensa. CIDH manifiesta su preocupación por la prevalencia de asesinatos y otras formas de violencia extrema contra las mujeres en Perú. Recuperado de: https://www.oas.org/es/cidh/prensa/ comunicados/2018/144.asp

- Comisión Interamericana de Derechos Humanos (2019). Violencia y discriminación contra mujeres, niñas y adolescentes: Buenas prácticas y desafíos en América Latina y en el Caribe. Anexo 2 Impactos de casos de discriminación y violencia contra mujeres, niñas y adolescentes. 
Recuperado de: http://www.oas.org/es/cidh/informes/pdfs/violencia- discriminacion-mujeresAnexo2-es.pdf

- Corte Interamericana de Derechos Humanos (2009). Sentencia González y otras (Campo Algodonero) vs. México. Recuperado de: https://www.corteidh.or.cr/docs/casos/articulos/ seriec_205_esp.pdf

- Corte Interamericana de Derechos Humanos (2018). Cuadernillo de Jurisprudencia de la Corte Interamericana de Derechos Humanos. Recuperado de: https://www.corteidh.or.cr/sitios/libros/ todos/docs/cuadernillo4.pdf?fbclid=IwAR2HYPKRUcC XGiGrRHLc49Kn9GkpdW3da0j_ B10IYbiNGAq_miATVLFUtwg

- Instituto Nacional de Estadística e Informática (2018). Capítulo 12. Violencia contra las mujeres, niñas y niños. Recuperado de: https://www.inei.gob.pe/media/MenuRecursivo/ publicaciones digitales/Est/Lib1656/index1.ht ml

- Ministerio de la Mujer y Poblaciones Vulnerables (2016). Violencia basada en género. Marco conceptual para las políticas públicas y la acción del Estado. Lima: MIMP.

- Ministerio de la Mujer de Paraguay (S/F). Ministra integró panel internacional sobre violencia y pandemia. Recuperado de: http://www.mujer.gov.py/index.php/noticias/ministra-de-la- mujerintegro-panel-internacional-sobre-violencia-contra-mujeres-y-pandemia

- Ministerio de la Mujer y Equidad de Género de Chile (2020). Plan de contingencia de atención, protección y reparación ante los efectos del coronavirus. Recuperado de: https:// www.sernameg.gob.cl/?page_id=31045

- Ministerio de la Mujer y Poblaciones Vulnerables (2009). Plan Nacional contra la Violencia Hacia la Mujer: 2009 - 2015. Recuperado de: https://www.mimp.gob.pe/contigo/contenidos/ pncontigo-articulos.php?codigo=7

- Ministerio de la Mujer y Poblaciones Vulnerables (2019). Cartilla Alerta de Seguridad. Recuperado de: https://repositoriopncvfs.pe/wp-content/uploads/2019/05/Alerta-deseguridad.pdf

- Movimiento Manuela Ramos (2011). Argumentos para el debate sobre la tipificación del feminicidio. Recuperado de: http://www2.congreso.gob.pe/Sicr/TraDocEstProc/ Contdoc01_2011.nsf/d99575da99ebfbe30 5256f2e006d1cf0/86f083733df2572e052579b200 6b7e1b/\$FILE/OFV08300911.pdf

- Perú 21 (2020). Abogado de presuntos violadores indigna con su frase: "A la señorita le gustaba la vida social. Recuperado de: https://peru21.pe/lima/abogado-de-presuntosvioladores-de-joven-de-21-anos-a-la-senorita-le-gustaba-la-vida-social-video-surco-noticia/

- Tribunal Constitucional del Perú. (2020). STC Exp. № 03378-2019-PC/TC. Recuperado de: https://tc.gob.pe/jurisprudencia/2020/03378-2019-AA.pdf

Fecha de recepción: 23 de octubre de 2020

Fecha de aceptación:06 de noviembre de 2020 